4311

Inflammatory biomarkers of cognitive dysfunction in pediatric obesity: associations between Executive Function, C-Reactive Protein, Interleukin 6, and Tumor Necrosis Factor alpha

Kathryn Prendergast ${ }^{1}$, Caroline Keller, MPH, Shima Dowla, PhD, and Marissa Gowey, PhD

${ }^{1}$ University of Alabama at Birmingham

OBJECTIVES/GOALS: Executive function (EF) deficits lead to poorer adherence and weight loss in obesity treatment. Conversely, untreated obesity leads to both $\mathrm{EF}$ impairments and risk of chronic disease. $\mathrm{EF}$ deficits in children with obesity have begun to be associated with biomarkers of chronic disease risk such as glucose and cortisol. Elevated pro-inflammatory biomarkers, such as C-Reactive Protein (CRP), Interleukin 6 (IL-6), and Tumor Necrosis Factor a (TNFa), indicate risk for obesity-associated chronic disease and may represent another candidate biomarker of EF deficits in pediatric obesity. These inflammatory markers tend to be elevated in obesity but have yet to be examined in association with EF in pediatric obesity. This represents an opportunity to identify potential biomarkers of $\mathrm{EF}$ which may serve as novel treatment targets that could improve outcomes and chronic disease prevention. Thus, the present study aims to explore associations between EF and blood serum CRP, IL-6, and TNFa. METHODS/ STUDY POPULATION: Treatment-seeking children aged 8-12 years with $\mathrm{BMI}>95^{\text {th }}$ percentile were recruited from a pediatric weight management clinic and attended baseline assessments for a familybased behavioral intervention program for pediatric obesity. Demographics and medical history were assessed via parent questionnaire. Height and weight were measured by research staff and converted to zBMI. Child performance-based EF was assessed via the $\mathrm{NIH}$ Toolbox Cognitive Battery iPad application. Blood draws, assays, and Dual-energy X-ray Absorptiometry (DXA; measuring adiposity) were conducted by trained research personnel. Pearson's correlations were conducted to explore associations between EF (NIH Toolbox Cognitive Battery, fully corrected T-scores) and CRP, IL-6, and TNFa. RESULTS/ANTICIPATED RESULTS: Children $(\mathrm{n}=12)$ were primarily female (76.5\%) and African American (64.7\%). Correlation coefficients between inflammatory markers and $\mathrm{EF}$ were highly variable $(r=-0.39$ to 0.52$)$. CRP showed small, negative associations with Cognitive Flexibility $(r=-0.39)$ and Inhibitory Control $(r=-0.34)$. IL-6 also demonstrated a small, negative association with Inhibitory Control $(r=-0.37)$. TNFa was positively and moderately associated with working memory $(\mathrm{r}=0.53)$. Remaining associations were weak $(-0.3<\mathrm{r}<0.3)$. DISCUSSION/SIGNIFICANCE OF IMPACT: Signals of higher inflammation, measured via CRP and IL-6, in association with EF deficits were identified in a small sample of children with obesity, as hypothesized. However, signals in the opposite direction were identified as well, measured via TNFa. CRP and IL-6 may represent candidate biomarkers of executive dysfunction in obesity that warrant further domain and biomarker-specific research, with potential long-term implications for improving pediatric obesity treatment.

4091

Influence of Vision and Proprioception on Motor Control in ASD

Robin L Shafer ${ }^{1}$, Zheng Wang ${ }^{2}$, and Matthew W. Mosconi ${ }^{1}$

${ }^{1}$ University of Kansas; ${ }^{2}$ University of Florida

OBJECTIVES/GOALS: Sensorimotor integration deficits are common in Autism Spectrum Disorders (ASD). There is evidence for both an over-reliance on visual and proprioceptive feedback during motor control in ASD, suggesting deficits in the ability to modulate sensory feedback processing in order to use the most reliable input. This study aims to test this hypothesis. METHODS/STUDY POPULATION: 40 persons with ASD (ages 10-33 yrs) and 25 age-, sex- and nonverbal IQ-matched controls completed precision gripping tasks under multiple proprioceptive and visual feedback conditions. Participants squeezed a force sensor with their index finger and thumb and tried to match their force output to a target force. Visual feedback of the target force (stationary bar) and their force output (bar that moved up/down with increased/decreased force) were displayed on a computer screen. Visual feedback was presented across low, medium, and high gain levels; the force bar moved a greater distance per change in force at higher gains. Proprioceptive feedback was manipulated using $80 \mathrm{~Hz}$ tendon vibration at the wrist to create an illusion that the muscle is contracted. Force regularity (approximate entropy; ApEn) was examined. RESULTS/ANTICIPATED RESULTS: We have scored data from 18 participants with ASD and 13 control participants to date. Preliminary results from these participants indicate a Group $\mathrm{x}$ Tendon Vibration $\mathrm{x}$ Visual Gain interaction for ApEn ( $\mathrm{F}=1.559$, $\mathrm{p}$ $=0.023$ ). Individuals with ASD show slight increases in ApEn with $80 \mathrm{~Hz}$ tendon vibration relative to no tendon vibration in all visual conditions. Controls showed increased ApEn during $80 \mathrm{~Hz}$ compared to no tendon vibration at low visual gain but decreased ApEn with tendon vibration at high visual gain. These preliminary results indicate that controls shift to using a secondary source of sensory feedback (e.g., proprioception) when the primary source (e.g., vision) is degraded. However, persons with ASD do not reweight different sensory feedback processes as feedback inputs are degraded or magnified. DISCUSSION/SIGNIFICANCE OF IMPACT: Our preliminary results reveal that sensorimotor issues in ASD result from deficits in the reweighting of sensory feedback. Namely, persons with ASD fail to dynamically recalibrate feedback processes across visual and proprioceptive systems when feedback conditions change. Our results may aid treatment development for sensorimotor issues in ASD.

4261

Insulin Sensitizing Effects of Vitamin D Mediated through Reduced Adipose Tissue Inflammation and Fibrosis: Evidence from a Human Randomized Trial and Mice Studies

Eric Lontchi Yimagou ${ }^{1}$, Sona Kang ${ }^{2}$, Kehao Zhang ${ }^{1}$, Akankasha Goyal $^{1}$, Jee Young You ${ }^{1}$, Evan Rosen ${ }^{3}$, Preeti Kishore ${ }^{1}$, and Meredith Hawkins $^{1}$

${ }^{1}$ Albert Einstein College of Medicine; ${ }^{2}$ University of California, Berkeley; ${ }^{3}$ Harvard Medical School

OBJECTIVES/GOALS: Vitamin D [25(OH)D], known to have antiinflammatory and anti-fibrotic effects in other tissues, may also impact adipose tissue. We designed parallel studies in humans and rodents to define the effects of vitamin $\mathrm{D}$ on adipose tissue inflammation and fibrosis, and on systemic insulin resistance. METHODS/STUDY POPULATION: We performed a randomized, double-blinded placebo-controlled trial to examine the effects of repleting vitamin $\mathrm{D}$ at to two levels (to $>30 \mathrm{ng} / \mathrm{ml}$ and to $>50 \mathrm{ng} / \mathrm{ml})$ in $25(\mathrm{OH}) \mathrm{D}$-deficient $(<20 \mathrm{ng} / \mathrm{ml})$, insulin resistant, overweight-to-obese humans $(\mathrm{n}=19)$. A comprehensive study of whole-body insulin action was undertaken with euglycemic stepped 
hyperinsulinemic clamp studies, both before (1st visit) and after administration of vitamin $\mathrm{D}$ or placebo (2nd visit and $3 \mathrm{rd}$ visit). Adipose tissue fibrosis and inflammation were quantified by 'realtime' rt-PCR and immunofluorescence. To determine whether vitamin D's effects are mediated through adipocytes, we performed hyperinsulinemic clamp studies and adipose tissue analysis in an adipocyte-specific vitamin D receptor knockout (VDR KO) mouse model. RESULTS/ANTICIPATED RESULTS: $25(\mathrm{OH}) \mathrm{D}$ repletion (to $>30 \mathrm{ng} / \mathrm{ml}$ ) was associated with reductions in adipose tissue expression of inflammatory (0.6-0.7-fold decreased expression of TNF- $\alpha$, IL-6, iNOS and PAI-1) and pro-fibrotic (0.4-0.8-fold decreased expression of TGF- $\beta 1$, HiF1 $\alpha$, Collagen I, V, VI and MMP7) factors, decreased collagen VI immunofluorescence ( $p$ $=0.02$ ) and improved hepatic insulin sensitivity in humans, with suppression of endogenous glucose production (EGP) $(1.28 \pm 0.20$ vs $0.88 \pm 0.18 \mathrm{mg} / \mathrm{kg} / \mathrm{min}, \mathrm{p}=0.03)$. Compared to wild type (WT), VDR KO mice exhibited increased adipose tissue expression of several pro-inflammatory (Tnf- $\alpha$, iNos, Pai-1, Mcp- 1 and F4/80; 4-10 fold) and pro-fibrotic genes (Tgf- $\beta 1$, Collagen VI, and Tsp1; 2-4 fold), in concert with hepatic insulin resistance (EGP $10 \pm 3$ vs $3 \pm 2 \mathrm{mg} / \mathrm{kg} / \mathrm{min}$ in WT, $\mathrm{p}=0.021$ ). DISCUSSION/SIGNIFICANCE OF IMPACT: Collectively, these complementary human and rodent studies establish a beneficial role of vitamin $\mathrm{D}$ to improve hepatic insulin resistance, likely by restraining adipose tissue inflammation and fibrosis. Thus, normalizing 25(OH)D levels could have metabolic benefits in targeted individuals. CONFLICT OF INTEREST DESCRIPTION: N/A

4329

Investigating the role of Klotho in neurocognitive outcomes, brain volumes, and white matter changes in pediatric brain tumor survivors

Caleb Simpeh Edwards ${ }^{1}$, Schuyler Stoller, Sol Savchuk, Christian Rodrigo Ugaz Valencia, Liz Tong, Andreas Rauschecker, Dena Dubal, Cassie Kline, and Sabine Mueller

${ }^{1}$ University Of California, San Francisco

OBJECTIVES/GOALS: Klotho is a protein linked to improved cognition in aging adults. A specific KLOTHO gene variant, KL-VS, increases circulating levels of Klotho. The current study aims to identify if the KL-VS haplotype and Klotho levels are associated with improved neurocognition in pediatric brain tumor survivors. METHODS/STUDY POPULATION: We are actively accruing pediatric brain tumor patients at UCSF alongside an existing multi-institutional cohort study investigating radiation-induced vasculopathies and cognitive outcomes in this population. Normal controls are being enrolled in parallel. Each patient undergoes: 1) single nucleotide polymorphism genotyping to identify KL-VS haplotype status, 2) enzyme-linked immunosorbent assays to measure circulating Klotho, 3) neurocognitive assessments with a computer-based, validated Cogstate battery, and 4) brain volume and white matter lesion segmentation analyses using MRI sequences obtained as part of routine care. RESULTS/ANTICIPATED RESULTS: Genotyping has been performed on 99 enrolled patients. KL-VS heterozygosity was seen in $22.7 \%$ of patients. To date, KL-VS status is not associated with neurocognitive outcomes at baseline or Year 1 testing. Association between KL-VS status, circulating Klotho levels, neurocognitive outcomes, brain volume and white matter lesion segmentation analyses is ongoing. We hypothesize that elevated Klotho levels will be associated with improved neurocognition, increased brain volumes in regions of interest and decreased white matter lesion volumes. DISCUSSION/SIGNIFICANCE OF IMPACT: If circulating Klotho leads to improved neurocognition in pediatric brain tumor survivors, Klotho levels might serve as a prognostic biomarker. Furthermore, as Klotho is being investigated for therapeutic indications, it may represent an intervention to prevent cognitive deficits in these patients.

4198

Investigating the Role of Rab27B in Non-Small Cell Lung Cancer Tumor Initiating Cells

Danielle Beetler ${ }^{1}$, Kayleah Beltran ${ }^{1}$, Kayla Lewis ${ }^{1}$, and Verline Justilien $^{1}$

${ }^{1}$ Mayo Clinic

OBJECTIVES/GOALS: Rab27B, a small GTPase, functions in exosome formation and secretion. Rab27B is overexpressed in non-small cell lung cancer (NSCLC) and predicts patient survival; however, little is known about its importance in NSCLC cells. Here, we investigated the role of Rab27B in NSCLC tumor initiating cells. METHODS/STUDY POPULATION: Tumor initiating cells (TICs) were enriched in a panel of NSCLC cell lines using low adherence spheroid cultures. QPCR and immunoblot analysis were used to compare Rab27B mRNA and protein expression, respectively, in adherent bulk cancer cells and TIC cultures. Lentiviral-packaged short hairpin RNAs (shRNAs) were used to knockdown Rab27B in PC9 and H1299 NSCLC TICs. The effects of Rab27B knockdown on PC9 and H1299 TIC expansion, transformed growth, and invasion were analyzed by MTT cell proliferation, soft agar colony formation, and Boyden chamber assays respectively. RESULTS/ ANTICIPATED RESULTS: Quantitative PCR and immunoblot analysis showed that Rab27B expression is elevated in NSCLC TICs when compared to adherent bulk cancer cells. Efficient knockdown of Rab27B was achieved in PC9 and H1299 NSCLC TICs using two independent shRNA constructs. Rab27B knockdown cells exhibited decreased expansion as spheroid cultures, transformed growth, and invasion when compared to non-target shRNA control cells. Future experiments will focus on determining the importance of Rab27B in TIC exosome production and in vivo tumor growth and metastasis. DISCUSSION/SIGNIFICANCE OF IMPACT: Our results show that Rab27B is important in NSCLC TIC growth and invasion. Further studies are needed to determine the mechanism of Rab27B action. TICs have been linked to enhanced tumorigenic properties, suggesting that Rab27B could be a good candidate for therapeutic targeting of NSCLC TICs.

4249

\section{Markers of mitochondrial biogenesis, fusion and architecture are disturbed in PBMC from war veterans with posttraumatic stress disorder (PTSD)}

Silvana Andric ${ }^{1}$, Aleksandra Markovic ${ }^{2}$, Mirko Milosevic ${ }^{2}$, Sava Radovic ${ }^{2}$, Isidora Starovlah², Jelena Brkljacic ${ }^{3}$, Danijela Vojnovic Milutinovic ${ }^{3}$, Gordana Matic ${ }^{3}$, and Tatjana Kostic ${ }^{2}$

${ }^{1}$ LaRES/ChronAge, CeRES, Faculty of Sciences, University of Novi Sad; ${ }^{2}$ LaRES/ChronAge, Faculty of Sciences, University of Novi Sad; ${ }^{3}$ IBISS, University of Belgrade

OBJECTIVES/GOALS: The aim of this study was to define the transcription profiles of the molecular markers of mitochondrial biogenesis and fusion/architecture, and the markers of mtDNA copy numbers in the peripheral blood mononuclear cells (PBMCs) from 